\title{
ESTUDIOS SOBRE CONSISTENCIA EN LAS IDEAS DE LOS ALUMNOS EN CIENCIAS
}

\author{
OLIVA MARTINEZ, J. M. \\ Instituto de Bachillerato Fuerte de Cortadura, Vía Augusta Julia, s/n. Cádiz.
}

\section{SUMMARY}

This article reviews the different lines of research related to the studies about «consistency» in the ideas as expressed by students, a highly interesting concept both from the theoretical and practical standpoints.

\section{INTRODUCCIÓN}

Sin duda alguna, dejando aparte el estudio descriptivo de las ideas de los estudiantes en distintos dominios de conocimiento, uno de los problemas que más ha llamado la atención de los investigadores ha sido el de consistencia interna o no que presentan tales formas de pensamiento a través de distintos contextos. No en vano, dicha cuestión ha sido considerada de enorme importancia de cara a comprender la génesis y evolución de tales ideas, así como por su indudable interés educativo (Engel Clough y Driver, 1986; Pozo, 1987; Pozo et al., 1991; Pozo, Limón y Sanz, 1991). Prueba del interés que ostenta esta temática desde un punto de vista teórico, lo tenemos en el hecho de que ya la teoría piagetiana fuera objeto de múltiples críticas debido a la indeterminación observada en el uso de esquemas formales a través de los llamados «desfases» de razonamiento. Dada la trascendencia del tema y de las implicaciones teóricas y prácticas que del mismo se podrían derivar, el presente trabajo se dedica a mostrar una revisión bibliográfica de estudios desarrollados en esta dirección.

A lo largo del artículo, por consistencia entendemos la mayor o menor tendencia que presentan los alumnos y sus explicaciones para aportar ideas y concepciones semejantes a través de contextos o situaciones diferentes. Desde nuestro punto de vista, ese término es el que mejor se adecua al tema que nos ocupa en el presente trabajo, ya que la denominación de coherencia, que a veces también se le ha dado, nos parece más apropiada para aludir al grado interno de estructuración lógica de un razonamiento, o también al nivel de congruencia que el mismo presenta con las evidencias empíricas disponibles. Por su parte, reservaremos Ia denominación de estabilidad -término que también emplearemos- para hablar del grado en el que una determinada concepción tiende a aparecer en un mismo contexto o situación en dos momentos u ocasiones diferentes. Este término se corresponde, por una parte, con la fiabilidad de los resultados aportados por los instrumentos diagnósticos empleados y, por otra, también, con la dificultad con que estos esquemas cambian a través de la instrucción, es decir, con la viabilidad del cambio conceptual. La estabilidad se refiere, pues, también a un tipo especial de consistencia, si bien aquí la invarianza en las concepciones puestas en juego depende de un factor de naturaleza temporal y no de tipo contextual.

\section{ENFOQUES Y METODOLOGÍASEMPLEADAS}

Algunos de los estudios desarrollados en este ámbito tienen carácter empírico (Engel Clough y Driver, 1986; 
De Posada y Prieto, 1989; Finegold y Gorsky, 1991; entre otros). Se trata de trabajos en los que, primero, se recoge información acerca del alumno empleando algún tipo de instrumento, analizándose luego la convergencia observada entre los resultados obtenidos en situaciones diversas en las que se modifica el contexto involucrado. Otros trabajos tienen, en cambio, un carácter más bien reflexivo y teórico (Hewson, 1990; Pozo et al., 1991), dedicándose a discutir las posibles causas en las que subyace la mayor o menor variabilidad detectada en los esquemas de pensamiento usados. Son trabajos que suelen apoyarse en una sólida fundamentación teórica, a partir de la cual poder explicar los datos procedentes de Ios análisis empíricos disponibles.

Las técnicas e instrumentos usados varían đe unos estudios a otros, en consonancia con lo que ocurre en la generalidad de trabajos que se realizan en el campo de la detección de ideas intuitivas y esquemas alternativos. Así, mientras Engel Clough y Driver (1986) utilizan entrevistas individuales, otros autores como Maloney (1988) o Jiménez y Fernández (1989) prefieren emplear tareas escritas.

Desde una perspectiva metodológica, entre los trabajos de tipo empírico que aparecen en la bibliografía se pueden distinguir dos grandes grupos. El primero de ellos abarca la mayoría de las referencias encontradas al respecto, y en ellos se aborda el análisis de la consistencia a un nivel grupal sin liegar apenas a emplear diseños estadísticos exhaustivos que permitan trazar conclusiones de alto nivel. Se trata normalmente de trabajos de carácter descriptivo, que implican la comparación de frecuencias de respuestas asignadas a cada uno de los esquemas delimitados en distintos ítems. Las diferencias entre esas frecuencias se toma como una medida de la inconsistencia observada, aplicándose en ocasiones pruebas estadísticas como el $\chi^{2}$ para analizar la significatividad estadística de las diferencias detectadas. Ejemplos de trabajos de este tipo lo encontramos en De Posada y Prieto (1989) o Maloney (1988).

En segundo lugar, y a raíz del trabajo de Engel Clough y Driver (1986), el panorama ha cambiado algo, observándose un desplazamiento hacia trabajos en los que se llega a investigar, alumno por alumno, la consistencia con la que cada uno pone en juego sus ideas a través de diferentes situaciones y contextos. En este caso, los estudios realizados resultan algo más complejos, analizándose en ellos las tipologías de respuestas que cada uno ofrece ante cuestiones diferentes, y comparándolas luego a través de tablas de frecuencias cruzadas o mediante análisis de fiabilidad y consistencia interna (Litch y Thifs, 1990; Finegold y Gorsky, 1991; Oliva y Rosado, 1990). Los cálculos estadísticos juegan aquí un carácter mucho más relevante, siendo frecuente eI uso de técnicas destinadas al análisis de tablas de contingencias o estudios de carácter factorial de tipo multivariante.

A continuación dedicamos espacio a discutir algunos de los resultados más relevantes que se desprenden de los estudios desarrollados en este terreno.

\section{RESULTADOS MÁS RELEVANTES EN ESTE TIPO DE ESTUDIOS}

\section{Sobre el nivel de consistencia de las ideas de los alumnos}

Una de las primeras referencias que encontramos sobre el tema, la tenemos en el trabajo de Champagne, Klopfer y Anderson (1980). En él ya se menciona que los alumnos suelen tener problemas a la hora de reconocer que las mismas leyes físicas son aplicables a objetos en caída libre y objetos que deslizan a través de planos inclinados. Por su parte, Clement (1982), al trabajar, con alumnos norteamericanos de nivel universitario, el problema de la asociación entre fuerza y movimiento y la representación de las fuerzas que actúan sobre objetos en reposo, observa que la dificultad de las tareas propuestas depende en cierta medida de la pregunta formulada. Resultados similares fueron obtenidos por Whitaker (1983) y Yates y otros (1988), quienes estudiaron diversas ideas sobre mecánica, observando que las frecuencias de contestaciones correctas dependen del contexto involucrado y también de la forma de plantear la preguntá. En esta misma línea se mueven los trabajos de Peters (1982), Cross y otros (1986) y Longden y otros (1991), quienes han detectado que en contextos de tipo formal se verifica una mayor evolución en las ideas que en contextos de tipo concreto o cotidiano. Ya en nuestro país podríamos citar los trabajos de Llorens (1987, 1988) o de Iglesias y otros (1990), quienes han detectado que los alumnos presentan una mayor tendencia al uso de modelos científicos -como el modeío discontinue de la materià- en cuestiones de opción múltiple que en cuestiones de respuesta abierta. También cabe citar el estudio de Sánchez y otros (1993), en el que se pudo comprobar cómo la familiaridad con el contexto fenomenológico en el que se desarrolla una tarea influye ostensiblemente en el grado en el que las ideas puestas en juego sobre composición de movimientos se mantienen arraigadas en la estructura cognitiva de los alumnos.

Por otro lado, Champagne, Gunstone y Klopfer (1985) observan que, tanto antes como después de la instruccion, los estudiantes of recen explicaciones que dependen de la situación específica considerada, sin que ni siquiera lieguen a ser capaces de percibir la necesidad de ser consistentes cuando se varía el contexto implicado. Dicha necesidad, en opinión de ciertos autores (Hewson, 1985), aunque constituiría una pauta de razonamiento esencial y característica para la comunidad científica, estaría ausente en el razonamiento de los estudiantes. Prueba de ello lo tenemos en los resultados obtenidos en trabajos como el de Halloun y Hestenes (1985), en el que se ha tratado de identificar, en una larga muestra de 4.000 estudiantes americanos, categorías conceptuales sobre física que fueran coherentes. Como conclusión deI mismo se obtiene que los estudiantes suelen hacer uso de categorías conceptuales híbridas, y se muestran inconsistentes al aplicar la misma noción en situaciones diferentes. Otros ejemplos, los encontramos en el trabajo de Watts (1983), en el cual se llega a aislar hasta ocho conceptos alternativos diferentes de fuerza empleados en situaciones diversas, o en el de Maloney (1988), en el 
que se observan diferencias importantes en la interpretación del movimiento oblicuo de un proyectil y el de un chorro de líquido. Este tipo de comportamiento inconsistente debe ser considerado lógico, ya que suele ser característico de las fases iniciales de aprendizaje de cualquier materia nueva, cuando sólo se dispone de un conjunto de ideas incompletas y desconectadas entre sí, más que de auténticos esquemas coherentes (Inhelder, Sinclair y Bovet, 1974; DiSessa, 1982).

Se plantea, a raíz de todo ello, la cuestión de en qué medida preexisten en la mente del alumno supuestos "esquemas alternativos» si, al fin y al cabo, las ideas que se ponen en juego poseen un importante grado de inestabilidad e inconsistencia. No podemos dar respuesta a esta cuestión sobre la única base de trabajos de tipo descriptivo como, fundamentalmente, son los que acabamos de mencionar en este apartado. Para ello hemos de recurrir a trabajos desarrolfados, también en esta línea, con un interés especial en el desarrollo de un soporte interpretativo y térico que dé sentido a los datos disponibles.

\section{SOBRE EL ESTATUS EPISTEMOLÓGICODE LAS CONCEPCIONES DE LOS ALUMNOS}

A la pregunta aludida podríamos plantear dos respuestas. Podríamos, por un Iado, suponer que esas ideas constituyen sólo artefactos originados a lo largo del propio proceso de investigación (McClelland, 1984), de manera que no se corresponderían en la práctica con ningún tipo de idea ligada a una estructura conceptual estable. Por el contrario, se podría argumentar que, si los alumnos no usan las mismas ideas a través de contextos diferentes, es porque los sujetos utilizan reglas superficiales en vez de esquemas más profundos a la hora de categorizar distintas situaciones físicas (Chi, Feltovich y Glaser, 1981; Reif, 1987; Veldhuis, 1987), o bien porque adoptan perspectivas locales para explicar los fenómenos abordados en vez de emplear criterios generales (Viennot, 1979; Engel Clough y Driver, 1986; Finegold y Gorsky, 1991), o quizás por ambas cosas a la vez (Hewson, 1990; Whitelock, 1991).

Con objeto de ilustrar el segundo punto de vista, el cual es el del que parecen mostrarse partidarios la mayor parte de los autores, comentaremos algunos de los trabajos que acabamos de citar. Por ejemplo, Reif (1987) condujo un estudio con la finalidad de llegar a explicar la inconsistencia observada en el pensamiento de los alumnos. Como resultado del mismo, encontró que, en una fase posterior al aprendizaje de temas de física, los estudiantes tendían a interpretar los fenómenos que se les presentaba evocando "fragmentos parciales» de conocimientos en lugar de hacerlo a partir de aspectos generales del mismo:

Cuando intentan aplicar un concepto, los estudiantes novatos raramente llegan a evocar la definición del concepto u otro aspecto general de conocimiento... En lugar de ello, recurren a diversos elementos especificos de conocimiento almacenados en sumemoria, los cuales recuperan y aplican sin una minima reflexión" (Reif, 1987, p. 316).

Por su parte, en el trabajo de Engel Clough y Driver se investiga la consistencia intercontextual de las ideas de los alumnos en el campo de la herencia, la conducción del calor y la presión en líquidos, notándose que, si bien en algunos casos se observan ideas definidas que se aplican en diversos contextos, en otros, la situación es más compleja, de forma que el contex to implicado induce a respuestas diferentes. Esto, debería interpretarse, según los propios autores, no tanto en términos de una ausencia de coherencia en el razonamiento de los alumnos, como en términos de una forma peculiar y diferente de categorizar las situaciones que se les plantea (Engel Clough y Driver, 1986). Ello, con frecuencia, suele hacerse atendiendo a características y rasgos que resu!tan inesperados:

Los resultados obtenidos en este estudio nos han llevado a replantearnos la idea de que aquellos estudiantes que no aplican los mismos preconceptos a contextos similares desde un punto de vista cientifico, no posean esque. mas alternativos sistemáticos... Debemos preguntamos cuáles son las situaciones a partir de las cuales los estudiantes construyen ideas similares, ya que es posible que los alumnos categoricen las distintas situaciones de acuerdo con criterios diferentes a los que emplean los cientificos» (Engel Clough y Driver, 1986, p. 489).

En esta misma línea se mueven las conclusiones del trabajo de Jiménez y Fernández (1989), en el cual se Ilega a detectar una asociación estadísticamente significativa entre las ideas de alumnos universitarios sobre el tema de la evolución biológica en dos contextos diferentes, a pesar de observarse también una cierta variabilidad en las respuestas ofrecidas. Cabría citar aquí, igualmente, el estudio llevado a cabo por Finegold y Gorsky (1991), en el que llegan a aislar algunas categorías conceptuales consistentes con relación a las fuerzas que actúan sobre objetos en movimiento, aunque también reconocen la existencia de un cierto grado de impredicibilidad de las creencias que se ponen en juego ante situaciones diversas.

Por su parte, Viennot, en un sugerente artículo publicado ya hace algunos años (Viennot, 1985), interpreta la variabilidad observada en las respuestas de los alumnos considerando que éstas se forman a partir de argumentos basados en reglas de inferencia del tipo si $[\ldots]$ entonces ...», que pueden aplicar o no en una ocasión determinada dependiendo del contexto involucrado. Si dos situaciones son caracterizadas como análogas, se aplicará con ellas la misma regla de inferencia, mientras que, si son caracterizadas de forma diferente, se hará con reglas distintas. En cualquier caso, el planteamiento que esta autora realiza implica que las ideas espontáneas puestas en juego no han de ser siempre consideradas, en sí mismas, las propias reglas causales de inferencia que se emplean en los razonamientos, sino sus manifestaciones 
externas mediatizadas por el contexto en el que se enmarca la pregunta. Además, Viennot postula que cuando las ideas implicadas se mueven en un terreno más lejano al de situaciones concretas, en las que los estudiantes carecen de una experiencia física previa, se recurriria a lo que denomina elementos hipotéticos mediadores que serían núcleos o formas de razonamiento intermedias entre las situaciones concretas planteadas y las respuestas ofrecidas. Dichos elementos trascenderían más allá de los contextos específicos y constituirían algo así como la base o esencia misma del pensamiento analógico. De hecho, ya Stavy (1990), en terminos piagetianos, ha llegado a interpretar la expansión del conocimiento como un proceso de transferencia por analogía llevada a cabo desde estímulos perceptibles inmediatos hasta situaciones similares carentes de componentes sensibles.

Tal perspectiva se encuentra proxima a la ofrecida en Ios trabajos de Pozo y otros (1991), en la cual se contempla la posibilidad de coexistencia en un mismo alumno de dos o más ideas diferentes, susceptibles de competir entre sí ante una situación determinada. De esta forma, la respuesta emitida se consideraría como el resultado de un proceso de decisión lógica, según ciertas normas, que iría precedido de una fase de activación de esquemas a partir de experiencias pasadas entre las que se buscarían ciertas pautas de similitud con las del problema en cuestión (Flick, 1991).

Ejemplo de ese tipo de reglas de decisión lo sería la accesibilidad, que constituiría la probabilidad de atribuir un efecto a una causa determinada. Según los autores citados, tendemos a atribuir los efectos a las causas más accesibles; es decir, a aquéllas que sean más fácilmente recuperables de nuestra memoria, proceso que se ve influenciado por tres tipos de factores (Pozo y otros):

a) Recencia: Tendemos a recuperar más fácilmente los datos e ideas que más recientemente hayan sido procesados.

b) Frecuencia: Suele recurrirse con más frecuencia a aquellas relaciones causales empleadas un mayor número de veces.

c) Saliencia: Suele recurrirse a criterios dominados por los aspectos más destacados y Ia información que más sobresale del campo perceptivo.

De esta manera, ciertos factores relacionados con la recencia del fenómeno en la experiencia sensorial del alumno, o con la de otro similar activado por analogía (Flick, 1991), podrían contribuir a explicar una cierta parte de la indeterminación observada en las ideas ante un mismo fenómeno en dos ocasiones diferentes. Por otro lado, factores relacionados con la saliencia del fenómeno planteado, entre los que podríamos destacar los aspectos figurativos del problema of factores relacionados con el lenguaje utilizado, podrían explicar igualmente buena parte de la variabilidad detectada ante cuestiones formuladas en contextos distintos.
En rejación con este tema podríamos citar también a Hewson (1990), quien señala que muchos estudiantes no buscan las características comunes esenciales, sino que parecen usar, más bien, formas de razonamiento escasamente parsimoniosas llevadas a cabo a partir de rasgos superficiales o sobre aspectos particulares. Las opiniones de Hewson se muestran coincidentes con las de otros antores ya citados (Viennot, 1985; Engel Clough y Driver, I986; Reif 1878; Finegold y Gorsky, 1991), a través de las cuales se mantiene que los estudiantes suelen presentar dificultades a la hora de identificar los elementos esenciales de un sistema y alcanzar a partir de ellas conclusiones adecuadas.

Una consecuencia del nulo uso de criterios tales como precisión, consistencia, generabizabilidad y parsimonia al evaluar sus propias concepciones es que dichas concepciones resultan ser de utilidad limitada. Suministran explicaciones de hechos especificos, pero no incluyen criterios para determinar qué otros hechos pueden o no requerir explicaciones parecidas. A menudo son incapaces de predecir resultados de hechos de tipos similares, y dichos fallos es más probable que conduzcan a una proliferación de concepciones separadas que a un cambio hacia una concepción más general. En resumen, parece que los estudiantes que no usan dichos criterios son incapaces de apreciar los beneficios que proceden de las teorías que -como la necánica newtoniana-los satisfacen" (Hewson, 1990, p. 160).

Se pone en evidencia, con claridad, la existencia de un gran paralelismo entre esas características del pensamiento intuitivo del alumno y las aportadas por otros autores, como Gil y Carrascosa (1990), para quienes el origen de estas nociones estaría en el uso de lo que ellos llaman «metodología de la superficialidad» a la hora de examinar el mundo que les rodea. Según esos autores, la metodología empleada llevaría a los estudiantes a realizar inferencias y generalizaciones «acríticas», tomando como base experiencias no controladas guiadas por el «sentido común».

Por otra parte, como se habrá podido ver a través de las palabras de Hewson, se ha llegado a asociar el problema de la consistencia en Ias ideas empleadas con el de las posibilidades de aprendizaje y de cambio conceptual. Según este punto de vista, la consistencia en el razonamiento constituiría una condición esencial en la comprensión de la mecánicá newtoniana (Minstrell, 1982), de forma que la toma de conciencia de cuáles son las similitudes y diferencias esenciales entre diversos fenómenos análogos constituiría un prerrequisito para la aceptación de nociones no dependientes del contexto, como lo son las teorías científicas (Hewson, 1990). En la misma línea se encuentran autores como Litch y Thijs (1990) o Gauld (1988), quienes consideran que aquellos estudiantes que muestran ideas alternativas consistentes a lo largo de diversos contextos presentarán también la habilidad de razonamiento que se requiere para la aceptación de las ideas científicas. Ello, según algunos investigadores, supondría que una idea alternativa consistente podría ser considerada como una etapa intermedia en el cambio conceptual. Por ello, los alumnos no sólo debe- 
raan sentir necesidad de cambiar sus ideas, sino que, incluso, deberían desarrollar habilidades para generalizar y eliminar contradicciones (Gauld, 1988; Finegold y Gorsky, 1991). De hecho, parte de las dificultades del cambio podrían estar tanto en la propia asimilación de los aprendizajes propiamente dicha, como en la capacidad de trasladar las ideas que se poseen de unos contextos a otros (Oliva y Rosado, 1990; Pozo, Limón y Sanz, 1991) y en el uso de los conocimientos "adquiridos», en contextos familiares que se encuentran para el alumno muy alejados del formalismo académico (Pozo, Limón y San2, 1991).

\section{CONCLUSIONES Y EXPECTATIVAS FUTU-RAS EN TORNO AL TEMA}

Como vemos, a tenor de la actualidad de gran parte de las citas mencionadas, la temática se encuentra en este instante en un momento álgido, y ofrece un panorama de un alto interés tanto desde el punto de vista térico como práctico. Por el momento, los trabajos desarrollados en este ámbito han mostrado la existencia de niveles de consistencia variables, aunque significativos (Pozo et al., 1991), en los esquemas usados, lo que viene a indicar que tales formas de pensamiento son consideradas como más que simples nociones inventadas sobre la marcha. No obstante, se desprende de forma inmediata la necesidad de un mayor caudal de datos empíricos que completen y amplien el ya existente, sobre todo en relación con la significación estadística de la consistencia y estabilidad de las preconcepciones en diversas áreas.

\section{REFERENCIAS BIBLIOGRÁFICAS}

AUSUBEL, D. P., NOVAK, J. D. Y HANESIAN, H. (1983). Psicologia educativa. Un puntode vista cognoscitivo. México: Trillas.

CLEMENT, J. (1982), Student preconceptions in introductory mechanics. American Journal of Physics, 50(1), pp. 66-71.

CROSS, D., MAURIN, M., AMOROUX, R., CHASTRETTE, M., WEBER, J. y FAYOL, M. (1986). Conceptions of firstyear university students of the constituents of matter and the notions of acids and bases. European Journal of Science Education, 8, pp. 305-3\$3.

CHAMPAGNE, A.B., KLOPFER, L.E. y ANDERSON, J.H. (1980). Factor influencing the learning of classical mechanics. American Journal of Physics, 48, pp. 1074-1079.

CHAMPAGNE, A., GUNSTONE, R. y KLOPFER, L. (1985). Instructional consequences of students' knowledge about Physical Phenomena. Cognitive Science, 5, pp. 121-152.

CHI, M.T., FELTOVICH, P.J. y GLASER, R. (1981). Categorization and representation of physics problems by experts and novices. Cognitive Science, 5, pp. $121-152$.
También se pone de manifiesto la necesidad de un mayor número de trabajos interpretativos que expliquen ta variabilidad observada en las respuestas de los alumnos, en línea con los trabajos de Pozo y otros (1991). Precisamente, autores como Jiménez y otros (1994) consideran el estudio sobre la consistencia en las ideas de los alumnos como uno de los aspectos relevantes a tener en cuenta a la hora de perfilar el estatus epistemológico de las distintas concepciones y, por consiguiente, con vistas a delimitar en cada caso el tipo de denominación más adecuada que merece cada una de esas concepciones.

Por último, diremos que resulta necesario el desarrollo de esfuerzos tendentes a buscar estrategias didacticas que tomen en consideración los resultados de tales investigaciones, ya que, como señalan Ausubel y otros (1983), un mejor conocimiento acerca de cómo aprenden los alumnos y cómo influyen las variables de cambio podría repercutir directamente en la búsqueda de fórmulas tendentes a ayudarles a aprender mejor.

\section{AGRADECIMIENTOS}

Quisiera expresar mi agradecimiento a los(as) evaluadores(as) del presente artículo por las sugerencias y crfticas aportadas. Sin duda, los comentarios que de ellos he recibido han servido para enriquecer la version final del trabajo.

DI SESSA, A. (1982). Unlearning Aristotelian Physics: A study of knowledge-based learning. Cognitive Science, 6 , pp. 37.75 .

DEPOSADA,J.M. y PRIETO, T. (1989). Ideas y representaciones de los alumnos sobre radiactividad. Revista de Educaciónt. 289 , pp. 357-375.

DRIVER, R. (1986). Psicolgía cognoscitiva y esquemas conceptuales de los alumnos. Enseñanza de las Ciencias, 4(1), pp. 3-15.

ENGEL CLOUGH, E. y DRIVER, R. (1986). A study of consistency in the use of students'conceptual framework across different task contexts. Science Education, 70(4), pp. 473-493.

FINEGOLD, M. y GORSKY, P. (1991). Students' concepts of force as applied to related physical systems: a search for consistency. International Journal of Science Education, 13(1), pp. 97-113.

FLICK, L., (1991). Where concepts meet precepts: stimulating analogical thought in children. Science Education, 75(2), pp. $215-230$ 
GAULD, C.F, (1988). The cognitive context of pupils' alternative frameworks. International Journal of Science Education, 10(3), pp. 267-274.

(iIL, D. y CARRASCOSA, J. (1990). What to do with science «Misconceptions». Science Education, 74(5), pp. 53 I-540.

HALLOLN, A.I y HESTENES, D. (1985). The initial knowledge state of college physics students. American Journal of Physics, 53(11), pp. 1043-1055.

HEWSON, P.W. (1985). Epistemological commitments in the learning of science: Examples for dynamics. European Journal of Science Education, 7(2), pp. 163-172.

HEWSON, P.W. (1990). La enseñanza de "fuerza y movimiento" como cambio conceptual. Enseñanza de las Ciencias, 8(2), pp. 157-171.

IGLESIAS, A., OLIVA, J.M. y ROSADO, L. (1990). Las interacciones entre estudiantes en el trabajo en grupos y la construcción del modelo corpuscular de la materia y el principio de conservación de la masa. Investigación en la Escuela, 12, pp. 57-68.

INHEL,DER, B, , SINCLAIR, H. y BOVET, M. (1974). Leaming and the development of cognition. Harvard: Harvard University Press.

IIMÉNEZ, E., SOLANO, I. y MARÍN, N. (1994), Problemas de terminología en estudios reatizados acerca de «lo que el alumno ya sabe" sobre ciencias. Enseñanza de las Ciencias, 12(2), pp. 235-245.

JIMÉNEZ ALEIXANDRE, M.P. y FERNÁNDEZ, J. (1989). ¿Han sido seleccionados o se han acostumbrado? Ideas de estudiantes de biología sobre selección natural y consistencia entre ellas. Infancia y Aprendizaje, 47, pp. 67-81.

I.ITCH, P. y THIJS, G.D. (1990). Method to trace coherence and persistence of preconceptions. International Journal of Science Education, 12(4), pp. 403-416.

LONGDEN, K., BLACK, P. y SOLOMON, J. (1991). Children's interpretation of dissolving. International Journal of Science Educarion, 13(1), pp. 59-68.

LLORENS, J.A. (1987). Propuesta y aplicación de uno metodologia para analizar la adquisición de los conceptos químicos necesarios en la introducción a la teoria atomicomolecular: percepción de los hechos experimentales, sus representaciones y el uso dellenguaje en alumnos deformación profesional y bachillerato. Tesis doctoral no publicada. Universidad de Valencia.

LLORENS, J.A. (1988). La concepción corpuscular de la materia. Obstáculos epistemológicos y problemas de aprendizaje. Investigación en la Escuela, 4, pp. 33-48.

MALONEY, D.P. (1988). Novice rules for projectile motion. Science Education, 72(4), pp. 501-513.

POZO, J.I., (1987). Aprendizaje de las ciencias y pensamiento causal. Madrid: Visor

McCLlLLAND, J.A.G. (1984). Alternative frameworks: interpretation of cvidence. European Journal of Science Education, 6(1), pp. 1-6.
MINSTRELL, J. (1982). Explaining the «at rest» condition of an object. Physics Teacher, 20, pp. 10-14.

OL.IVA, J.M. y ROSADO, I. (1990). Influencia de la habiliclad de razonamiento formal y del estilo cognitivo en la naturaleza y consistencia de las ideas previas de los estudiantes sobre mecánica: primeros restultados, en Grupo de Investigación en la Escuela (comp.), Cambio cducativo y desarrollo profesional, pp. 252-262.

POZO, J.I., SANZ, A., GÓMEZ CRESPO, M.A. y I.IMÓN, M. (1991). Las ideas de los alumnos sobre la ciencia: una interpretación desde la psicología cognitiva. Eniseñana $d^{\prime}$ las Ciencias, 9(1), pp. 83-94.

POZO, J.I, SANZ, A., GÓMEZ CRESPO, M.A., LIMÓN, M. y SANZ, A. (1991). Procesos cognitivos en la comprension de la ciencia: las ideas de los adolescentes sobre la animica. Madrid: CIDE

POZO, J.I., LIMÓN, M. y SANZ, A. (1991). Conocimientos previos y aprendizaje escolar. Cuademos de Pedagogía, 188 , pp. $12-14$

REIF, F. (1987). Instructional design, cognition, and technology: Application to the teaching of scientific concepts. Journal of Research in Science Teaching, 24, pp. 309-324.

SÁNCHEL, L., OLIVA, J.M., ROSADO, L. y CRU\%, M.I. (1993). Detección de las ideas previas en cinemática utilizando la composición de movimicntos. Investigación en la Escuela, 19, pp. $105-116$

STAVY, J. (1990). Children's conceptios of change in state of matter: From liquid (or solid) to gas. Joumal of Reseurch in Science Teaching, 27, pp. 247-266.

VELDHCIS, G.H. (1987). Differences in the categorization of physics problems by novices and experts. Doctoral dissertation, lowa State University, (1986). Dissertation Abstratts International, 47(11), p. 3968-A.

VIENNOT, I. (1979). Spontaneous reasoning in elementary dynamics. European Journal of Science Fducation, 1(2), pp. $205-222$.

VIENNOT, L. ( 1985). Analysing students' reasoning in Science: A pragmatic view of theoretial problems. European Journal of Science Education, 7(2), pp. 151-162

WATTS, M. (1983). A study of schoolchildren's alternative fameworks of the concept of force. Europeat Joumal of Science Education, 5, pp. 217-230.

WHITAKLR, R.J. (1983a). Aristotle is not dead: student understanding of trajectory motion. American Joumal of Physics, 51, pp. 325-327.

WHITELOCK, D. (1991). Investigating a model of common sense thinking about causes of motion with 7 to 16 -year-old pupils. International Journal of Sciente Education, I3(3), pp. $321-340$.

YATES, J., BESSMAN, M., DUNNL, M., JERTSON, D. SKY, K. y NENDFLIIOE, B. (1988). Are conceptions of motion based on a naive theory or on prototypes? Cognition, 29 , pp. $251-275$. 